\title{
Selection of patients for intra-arterial treatment for acute ischaemic stroke: development and validation of a clinical decision tool in two randomised trials
}

\author{
Esmee Venema, , ${ }^{1,2}$ Maxim J H L Mulder, 2,3 Bob Roozenbeek, ${ }^{2}$ Joseph P Broderick, ${ }^{4}$ Sharon D Yeatts, ${ }^{5}$ \\ Pooja Khatri, ${ }^{4}$ Olvert A Berkhemer, ${ }^{3,6,7}$ Bart J Emmer, ${ }^{3}$ Yvo B W E M Roos, ${ }^{8}$ Charles B L M Majoie, ${ }^{6}$ \\ Robert I van Oostenbrugge, ${ }^{9}$ Wim $\mathrm{H}$ van Zwam, ${ }^{7}$ Aad van der Lugt, ${ }^{3}$ Ewout W Steyerberg, ${ }^{1,10}$ \\ Diederik W J Dippel, ${ }^{2}$ Hester F Lingsma'
}

\section{ABSTRACT}

OBJECTIVE

To improve the selection of patients with acute ischaemic stroke for intra-arterial treatment using a clinical decision tool to predict individual treatment benefit.

\section{DESIGN}

Multivariable regression modelling with data from two randomised controlled clinical trials.

\section{SETTING}

16 hospitals in the Netherlands (derivation cohort) and 58 hospitals in the United States, Canada, Australia, and Europe (validation cohort).

\section{PARTICIPANTS}

500 patients from the Multicenter Randomised Clinical Trial of Endovascular Treatment for Acute Ischaemic Stroke in the Netherlands trial (derivation cohort) and 260 patients with intracranial occlusion from the Interventional Management of Stroke III trial (validation cohort).

\section{MAIN OUTCOME MEASURES}

The primary outcome was the modified Rankin Scale (mRS) score at 90 days after stroke. We constructed an ordinal logistic regression model to predict outcome and treatment benefit, defined as the difference between the predicted probability of good functional outcome (mRS score 0-2) with and without intra-arterial treatment.

RESULTS

11 baseline clinical and radiological characteristics were included in the model. The externally validated $C$ statistic was 0.69 (95\% confidence interval 0.64 to ISRCTN10888758 (MR CLEAN).

\section{WHAT IS ALREADY KNOWN ON THIS TOPIC}

Intra-arterial treatment improves functional outcome in patients with acute ischaemic stroke caused by a proximal occlusion

In current practice there is large variation in the selection of candidates for intra-arterial treatment because of the uncertainty about treatment benefit in specific subgroups

\section{WHAT THIS STUDY ADDS}

A newly developed clinical decision tool combines multiple baseline clinical and radiological characteristics and shows large variations in treatment benefit between patients

Selection of individual patients for intra-arterial treatment should not be based on single patient characteristics

This model is the first step towards individualised selection of patients for intra-arterial treatment for acute ischaemic stroke and may be used as tool for assisting clinical decision making

0.73 ) for the ordinal model and 0.73 (0.67 to 0.79$)$ for the prediction of good functional outcome, indicating moderate discriminative ability. The mean predicted treatment benefit varied between patients in the combined derivation and validation cohort from $-2.3 \%$ to $24.3 \%$. There was benefit of intra-arterial treatment predicted for some individual patients from groups in which no treatment effect was found in previous subgroup analyses, such as those with no or poor collaterals.

\section{CONCLUSION}

The proposed clinical decision tool combines multiple baseline clinical and radiological characteristics and shows large variations in treatment benefit between patients. The tool is clinically useful as it aids in distinguishing between individual patients who may experience benefit from intra-arterial treatment for acute ischaemic stroke and those who will not.

TRIAL REGISTRATION

clinicaltrials.gov NCT00359424 (IMS III) and isrctn.com

\section{Introduction}

Stroke is the second most common cause of mortality worldwide and the most common cause of disability in high income countries. ${ }^{1}$ In Western countries, $80 \%$ of strokes are ischaemic. ${ }^{2}$ Ischaemic strokes caused by a proximal occlusion in the intracranial cerebral arteries result in poor outcome. ${ }^{34}$ Intra-arterial treatment improves functional outcome in patients with acute ischaemic stroke caused by a proximal occlusion, ${ }^{5-11}$ with a number needed to treat of 5 (odds ratio 2.35, 95\% confidence interval 1.85 to 2.98). ${ }^{12}$ However, this is an average treatment effect and it is likely that treatment benefit will vary for individual patients. ${ }^{1314}$ In current practice there is debate on the selection of candidates for intra-arterial treatment because of uncertainty of treatment benefit in specific subgroups and patients not included in the trials. ${ }^{1215}$

Clinicians combine multiple characteristics in their clinical decision making when treating an individual patient. For example, consider a man aged 70 who is admitted 40 minutes after the onset of symptoms, with a severe left hemisphere ischaemic stroke and a National Institutes of Health Stroke Scale (NIHSS) score of 22, an Alberta Stroke Program Early Computed Tomography Score (ASPECTS, see box 1) of 7, and an M1 occlusion but no collaterals on computed 


\section{Box 1: Descriptions of ASPECTS and collateral score}

\section{ASPECTS}

A quantitative grading system to assess early ischaemic changes on a non-contrast CT scan. Scores ranges from 0 to 10 , with 10 points for a normal CT scan and 1 point subtracted for every defined region with evidence of early ischaemic changes ${ }^{16}$

\section{Collateral score}

A 4 point scale to grade the collateral flow of the occluded territory on vessel imaging, with 0 representing absent collateral flow, 1 representing poor collateral flow $(<50 \%$ filling), 2 representing moderate collateral flow (between $50 \%$ and $100 \%$ filling), and 3 representing good collateral flow $(100 \% \text { filling })^{17}$

tomography (CT) angiography (see box 1). A previous subgroup analysis using data from the Multicenter Randomised Clinical Trial of Endovascular Treatment for Acute Ischaemic Stroke in the Netherlands (MR CLEAN) trial suggested no treatment effect for patients with no or poor collaterals. ${ }^{15}$ If this man can be treated soon after onset of stroke, will he benefit from intra-arterial treatment? Consider a woman aged 80 with diabetes and high systolic blood pressure, who arrived in a primary stroke centre too late for treatment with intravenous tissue plasminogen activator, with a NIHSS score of 22, ASPECTS of 9, and a carotid $\mathrm{T}$ occlusion with good collaterals on CT angiography. Should she be transferred to an intervention centre 40 miles away if intra-arterial treatment is just possible within the six hour time window?

We developed and validated a clinical decision tool to provide individualised predictions of the effect of intra-arterial treatment based on multiple characteristics. Such a tool may be helpful to support clinical judgment when making complicated decisions on intra-arterial treatment.

\section{Methods}

In short, we developed a multivariable prediction model in patients included in the MR CLEAN trial (Multicenter Randomised Clinical Trial of Endovascular Treatment for Acute Ischaemic Stroke in the Netherlands, $\mathrm{n}=500$ ) and validated this model in a subgroup of patients with an occlusion on CT angiography in the IMS III trial (Interventional Management of Stroke III, $\mathrm{n}=260$ ). The primary outcome was the modified Rankin Scale (mRS) score at 90 days after stroke. We constructed an ordinal logistic regression model to predict functional outcome and treatment benefit. This benefit was defined as the difference between the predicted probability of good functional outcome (mRS score 0-2) with and without intra-arterial treatment. Variables were selected using univariable and multivariable selection steps $(\mathrm{P}<0.15)$.

\section{Derivation cohort}

We used data from all 500 patients of MR CLEAN (derivation cohort) for the development of our model. ${ }^{5} \mathrm{MR}$ CLEAN was a phase III multicentre clinical trial with randomised treatment group assignment, open label treatment, and blinded outcome evaluation. Intraarterial treatment plus usual care was compared with usual care alone (control group). Usual care could include intravenous tissue plasminogen activator if eligible. Enrolled patients were 18 years or older (no upper age limit), had a score of 2 or higher on the National Institutes of Health Stroke Scale (NIHSS) (range 0-42), an occlusion of the proximal internal carotid artery, middle cerebral artery (M1 or M2), or anterior cerebral artery (A1 or A2), established with CT angiography. The start of intra-arterial treatment had to be possible within six hours after stroke onset. The imaging committee evaluated the findings on baseline non-contrast CT for the ASPECTS and non-invasive baseline vessel imaging (CT angiography, magnetic resonance angiography, or digital subtraction angiography) for the location of the occlusion and collateral score.

More detailed information about MR CLEAN can be found in the study protocol and the publication of the main results. 518

\section{Model development}

Patient characteristics obtained before treatment that are expected to predict outcome or to interact with treatment, based on expert opinion or recent literature, were specified in advance in our statistical analysis plan. ${ }^{19}$ We used ordinal logistic regression modelling, which assumes proportional odds, to test the effect of age, baseline NIHSS score, systolic blood pressure, treatment with intravenous tissue plasminogen activator, history of ischaemic stroke, atrial fibrillation, diabetes mellitus, pre-stroke mRS score, ASPECTS, location of occlusion, collateral score, and time to treatment, as well as the corresponding interactions with treatment. The primary outcome was the mRS score, a 7 point scale ranging from 0 (no symptoms) to 6 (death), at 90 days after stroke. ${ }^{20}$ For additional analyses, we derived the probabilities for good functional outcome (mRS score 0 -2) from the ordinal model. Treatment benefit was defined as the difference between the predicted probability of good functional outcome with and without intra-arterial treatment.

In our final multivariable model we selected the main effects or interaction terms with a $P$ value of $<0.15$ in univariable and multivariable analyses. Location of occlusion was analysed categorically and ASPECTS and collateral score were analysed continuously. Continuous variables were not dichotomised. Non-linearity of continuous variables was tested with restricted cubic spline functions. ${ }^{21}$ In the final model we used restricted cubic spline functions for age and systolic blood pressure. As a measure of time to treatment we used the time from stroke onset to groin puncture. Since groin puncture was not performed in control participants, time to groin puncture was not observable in the control arm. Single imputation based on regression using age, NIHSS score, transfer between hospitals, hospital of first presentation, and time to randomisation, was used to assign time to expected groin puncture $\left(R^{2}=0.89\right)$. Since all other variables were more than $98 \%$ complete within the derivation cohort, we used simple imputation by the mean for continuous 
variables and simple imputation by the mode for categorical variables.

Internal validation with bootstrapping was used to estimate the degree of optimism in the final model. To correct for this optimism we reduced the regression coefficients using penalised regression. ${ }^{21} 22$ Coefficients of non-linear terms and interaction terms were reduced with a larger penalty than the main effects. ${ }^{22}$

\section{External validation}

External validation of our model was performed in the IMS III trial. ${ }^{23}$ The IMS III trial $(\mathrm{n}=656)$ was a phase III multicentre clinical trial with randomised treatment group assignment, open label treatment, and blinded outcome evaluation. The trial tested the approach of intravenous tissue plasminogen activator followed by endovascular treatment compared with standard intravenous tissue plasminogen activator. Further details on the methods used in the trial have been reported extensively. 2324

We included patients with proved occlusion in the anterior circulation on non-invasive vessel imaging and an available mRS score at 90 days in the validation cohort $(n=260)$. Missing collateral scores because of insufficient CT angiography imaging $(n=68)$ were replaced by single imputation with regression using age, history of diabetes mellitus, and presence of internal carotid T occlusion. Single imputation for time to groin puncture $(n=102$, primarily control patients) was performed using age, NIHSS score, time to randomisation, and transfer between hospitals. All other variables were more than $98 \%$ complete. Missing values were imputed with the mean for continuous variables or the mode for categorical variables.

Model performance in the validation cohort was expressed by discrimination and calibration. Discrimination was quantified with the concordance or $\mathrm{C}$ statistic, which varies between 0.5 for a non-informative model and 1 for a perfectly discriminating model. ${ }^{25} \mathrm{We}$ calculated the general C statistic of our ordinal model and an additional $\mathrm{C}$ statistic for the predictions of good functional outcome (mRS score 0-2).

Calibration refers to the level of agreement between predicted risks and observed outcome; this was assessed graphically with a validation plot for the prediction of good functional outcome (mRS score 0-2) expressed as calibration slope and intercept. The calibration slope describes the effect of the predictors in the validation sample versus the derivation sample, and is ideally equal to 1 . The intercept indicates whether predictions are systematically too high or too low, and should ideally be zero. ${ }^{26}$

After external validation, the regression coefficients were fitted on a dataset combining all patients in the derivation and validation cohort. To assess if our model could be used to select individual patients for intra-arterial treatment, we estimated the individual predictions for all 760 patients included in this combined dataset. We created a scatter plot with the predicted probabilities of good functional outcome
(mRS score 0-2) for these patients without intra-arterial treatment on the $\mathrm{x}$ axis and the predicted probabilities with intra-arterial treatment on the $y$ axis. We made additional plots for the predictions of patients with no or poor collaterals and patients with low ASPECTS, since prespecified subgroup analyses showed that these groups had no or limited benefit of treatment.

All statistical analyses were performed with R statistical software (version 3.2.2) and the rms library (version 4.4-0). The web application was developed with the R Shiny package (shiny version 0.13.0).

\section{Patient involvement}

No patients were involved in setting the research question or the outcome measures, nor were they involved in developing plans for design or implementation of the study. No patients were asked to advise on interpretation or writing up of results. There are plans to disseminate the results of the research to the relevant patient community.

\section{Results}

Table 1 shows that the baseline patient characteristics and important characteristics of workflow and outcome were similar between the derivation cohort $(n=500)$ and validation cohort $(n=260)$. The validation cohort was somewhat more homogeneous, by not including patients with baseline disability (premorbid mRS score 23) or patients not treated with intravenous tissue plasminogen activator.

Most variables were predictors of outcome (table 2). The strongest predictors in multivariable analysis were age $(\mathrm{P}<0.001)$, baseline NIHSS score $(\mathrm{P}<0.001)$, systolic blood pressure $(\mathrm{P}<0.001)$, history of ischaemic stroke $(\mathrm{P}=0.03)$, diabetes mellitus $(\mathrm{P}=0.02)$, pre-stroke mRS score $(\mathrm{P}=0.003)$, ASPECTS $(\mathrm{P}=0.001)$, location of occlusion $(\mathrm{P}=0.03)$, and collateral score $(\mathrm{P}<0.001)$. Interactions with relative treatment effect were found in univariable analysis for history of ischaemic stroke, atrial fibrillation, time to groin puncture, and collateral score (all $\mathrm{P} \leq 0.10$, fig 1). In the multivariable model, the effects of intra-arterial treatment were similar to the univariable analysis, with larger effects in patients without previous ischaemic stroke $(\mathrm{P}=0.07)$, patients with better collateral scores $(\mathrm{P}=0.07)$, and patients with shorter times to groin puncture $(\mathrm{P}=0.13)$. Atrial fibrillation was not significant in multivariable analysis as either a main effect $(\mathrm{P}=0.67)$ or interaction effect $(\mathrm{P}=0.27)$, and was therefore excluded from the model.

The final multivariable model included age, baseline NIHSS score, systolic blood pressure, treatment with intravenous tissue plasminogen activator, history of ischaemic stroke, diabetes mellitus, pre-stroke mRS score, ASPECTS, location of occlusion, collateral score, and time from stroke onset to groin puncture. We added terms representing the interaction between treatment and each of previous stroke, collateral score, and time to groin puncture. The internally validated $\mathrm{C}$ statistic for ordinal outcome was 0.74 without interaction terms and this increased to 0.75 by adding interaction with 


\begin{tabular}{|c|c|c|}
\hline Characteristics & $\begin{array}{l}\text { Derivation } \\
\text { cohort } \\
(n=500)\end{array}$ & $\begin{array}{l}\text { Validation } \\
\text { cohort } \\
(n=260)\end{array}$ \\
\hline Mean (SD) age (years) & $65(14)$ & $67(12)$ \\
\hline Men & $292(58)$ & $135(52)$ \\
\hline Baseline score (interquartile range) on National Institutes of Health Stroke Scale & $18(14-22)$ & $17(14-21)$ \\
\hline Mean (SD) systolic blood pressure $(\mathrm{mm} \mathrm{Hg})$ & $145(25)$ & $149(26)$ \\
\hline Treatment with intravenous tissue plasminogen activator & $445(89)$ & $260(100)$ \\
\hline Allocation to intra-arterial treatment & $233(47)$ & $174(67)$ \\
\hline \multicolumn{3}{|l|}{ Medical history: } \\
\hline Ischaemic stroke & $54(11)$ & $28(11)$ \\
\hline Atrial fibrillation & $135(27)$ & $89(35)$ \\
\hline Diabetes mellitus & $68(14)$ & $49(19)$ \\
\hline \multicolumn{3}{|l|}{ Pre-stroke mRS score: } \\
\hline 0 & $404(81)$ & $231(89)$ \\
\hline 1 & $50(10)$ & $22(8)$ \\
\hline 2 & $25(5)$ & $7(3)$ \\
\hline$\geq 3$ & $21(4)$ & 0 \\
\hline \multicolumn{3}{|l|}{ Imaging: } \\
\hline Alberta Stroke Program Early CT score (interquartile range) on non-contrast CT & $9(8-10)$ & $8(6-9)$ \\
\hline \multicolumn{3}{|l|}{ Location of occlusion on non-invasive vessel imaging: } \\
\hline Internal carotid artery with terminal segment & $138(28)$ & $66(25)$ \\
\hline M1 & $319(64)$ & $144(55)$ \\
\hline M2 & $39(8)$ & $50(19)$ \\
\hline A1 & $3(1)$ & 0 \\
\hline \multicolumn{3}{|l|}{ Minutes (interquartile range) to stroke onset: } \\
\hline Randomisation & $200(150-261)$ & $143(120-170)$ \\
\hline Groin puncture & $260(210-311)$ & $205(168-235)$ \\
\hline Reperfusion & $340(274-395)$ & $275(238-319)$ \\
\hline \multicolumn{3}{|l|}{ Outcome: } \\
\hline Recanalisation (mTICI 2B/3) & $116(59)$ & $69(45)$ \\
\hline \multicolumn{3}{|l|}{ mRS score at 90 days: } \\
\hline 0 & $7(1)$ & $27(10)$ \\
\hline 1 & $36(7)$ & $46(18)$ \\
\hline 2 & $84(17)$ & $39(15)$ \\
\hline 3 & $87(17)$ & $36(14)$ \\
\hline 4 & $133(27)$ & $44(17)$ \\
\hline 5 & $45(9)$ & $18(7)$ \\
\hline 6 (mortality) & $108(22)$ & $50(19)$ \\
\hline
\end{tabular}

treatment. The $\mathrm{C}$ statistic for good functional outcome was 0.79 .

\section{External validation}

Similar effects were found for most variables in the validation cohort except for systolic blood pressure, diabetes mellitus, and the interaction between history of ischaemic stroke and treatment effect. The externally validated C statistic was 0.69 (95\% confidence interval 0.64 to 0.73 ) for the ordinal model and 0.73 ( 0.67 to 0.79 ) for the prediction of good functional outcome (fig 2).

The expected benefit of intra-arterial treatment varied largely between patients in the combined derivation and validation cohort (fig 3 top graph). Mean predicted absolute treatment benefit was an $11.8 \%$ higher probability of mRS score 0-2 compared with the probability without intra-arterial treatment, and varied from $-2.3 \%$ to $24.3 \%$ between individual patients in the combined derivation and validation cohort. The individual predictions for patients with no or poor collaterals (score 0 or 1) or low ASPECTS (score 0-5) illustrate the substantial variation in outcome and treatment benefit in these groups (fig 3 middle and bottom graphs). For some patients, who have multiple characteristics that negatively affect treatment benefit, the model predicts no benefit or even harm.

We calculated the predicted probabilities of good functional outcome with and without intra-arterial treatment for the two patients described in the introduction (fig 4). The first patient is expected to benefit from intra-arterial treatment despite absent collaterals and moderate ASPECTS. The probability of achieving a good functional outcome increases by 11 percentage points, from $16 \%$ without intra-arterial treatment to $27 \%$ with intra-arterial treatment. The predictions for the second patient illustrate that a good collateral score does not guarantee a large treatment benefit. The 80 year old patient has a low probability of achieving a good functional outcome (3\% without intra-arterial treatment and 5\% with intra-arterial treatment), with some shift on the total mRS scale. 
Table 2 | Main effects in derivation cohort $(n=500)$

\begin{tabular}{|c|c|c|c|c|}
\hline \multirow[b]{2}{*}{ Variables } & \multicolumn{2}{|l|}{ Univariable model } & \multicolumn{2}{|l|}{ Multivariable model } \\
\hline & $\begin{array}{l}\text { Common odds } \\
\text { ratio* }(95 \% \mathrm{Cl})\end{array}$ & P value & $\begin{array}{l}\text { Common odds } \\
\text { ratio* }(95 \% \mathrm{Cl})\end{array}$ & Pvalue \\
\hline Intra-arterial treatment & 1.66 (1.21 to 2.28$)$ & 0.002 & 1.86 (1.34 to 2.59$)$ & $<0.001$ \\
\hline \multicolumn{5}{|l|}{ Age per year: } \\
\hline$<65$ & 0.97 (0.95 to 0.99$)$ & \multirow{2}{*}{$<0.001$} & $1.00(0.97$ to 1.02$)$ & \multirow{2}{*}{$<0.001$} \\
\hline$\geq 65$ & 0.92 (0.89 to 0.94$)$ & & 0.92 (0.89 to 0.95$)$ & \\
\hline Baseline National Institutes of Health Stroke Scale score per point & $0.91(0.88$ to 0.94$)$ & $<0.001$ & $0.93(0.90$ to 0.96$)$ & $<0.001$ \\
\hline \multicolumn{5}{|l|}{ Systolic blood pressure per $10 \mathrm{mmHg}$} \\
\hline$<130 \mathrm{~mm} \mathrm{Hg}$ & $1.12(0.88$ to 1.41$)$ & \multirow{2}{*}{$<0.001$} & $1.26(0.99$ to 1.61$)$ & \multirow{2}{*}{$<0.001$} \\
\hline$\geq 130 \mathrm{~mm} \mathrm{Hg}$ & $0.76(0.70$ to 0.83$)$ & & 0.77 (0.70 to 0.85$)$ & \\
\hline Treatment with intravenous tissue plasminogen activator & 1.85 (1.12 to 3.08$)$ & 0.02 & 1.62 (0.94 to 2.79$)$ & 0.08 \\
\hline History of ischaemic stroke & 0.48 (0.29 to 0.80$)$ & 0.005 & $0.53(0.31$ to 0.92$)$ & 0.03 \\
\hline Atrial fibrillation & 0.52 (0.36 to 0.73$)$ & $<0.001$ & $0.92(0.62$ to 1.36$)$ & 0.67 \\
\hline Diabetes mellitus & $0.37(0.23$ to 0.59$)$ & $<0.001$ & $0.55(0.33$ to 0.90$)$ & 0.02 \\
\hline Pre-stroke mRS score & $0.63(0.52$ to 0.77$)$ & $<0.001$ & 0.72 (0.58 to 0.90$)$ & 0.003 \\
\hline Alberta Stroke Program Early CT score per point & $1.16(1.07$ to 1.26$)$ & $<0.001$ & $1.16(1.06$ to 1.28$)$ & 0.001 \\
\hline \multicolumn{5}{|l|}{ Location of occlusion on non-invasive imaging: } \\
\hline Internal carotid artery with terminal segment & 1.0 (reference) & \multirow{3}{*}{0.02} & 1.0 (reference) & \multirow{3}{*}{0.03} \\
\hline M1 & 1.53 (1.08 to 2.17$)$ & & 1.43 (0.98 to 2.07$)$ & \\
\hline M2 & 2.11 (1.15 to 3.88$)$ & & 2.35 (1.20 to 4.60$)$ & \\
\hline Collateral score & $1.95(1.62$ to 2.36$)$ & $<0.001$ & $1.61(1.31$ to 1.96$)$ & $<0.001$ \\
\hline Time from stroke onset to groin puncture per 30 minutes & 0.94 (0.88 to 1.00$)$ & 0.07 & $0.93(0.86$ to 1.00$)$ & 0.04 \\
\hline
\end{tabular}

$\mathrm{CT}=$ computed tomography; $\mathrm{mRS}=$ modified Rankin Scale.

Presented common odds ratios reflect the effect on the reversed mRS.

*Value $>1$ corresponds to better functional outcome.
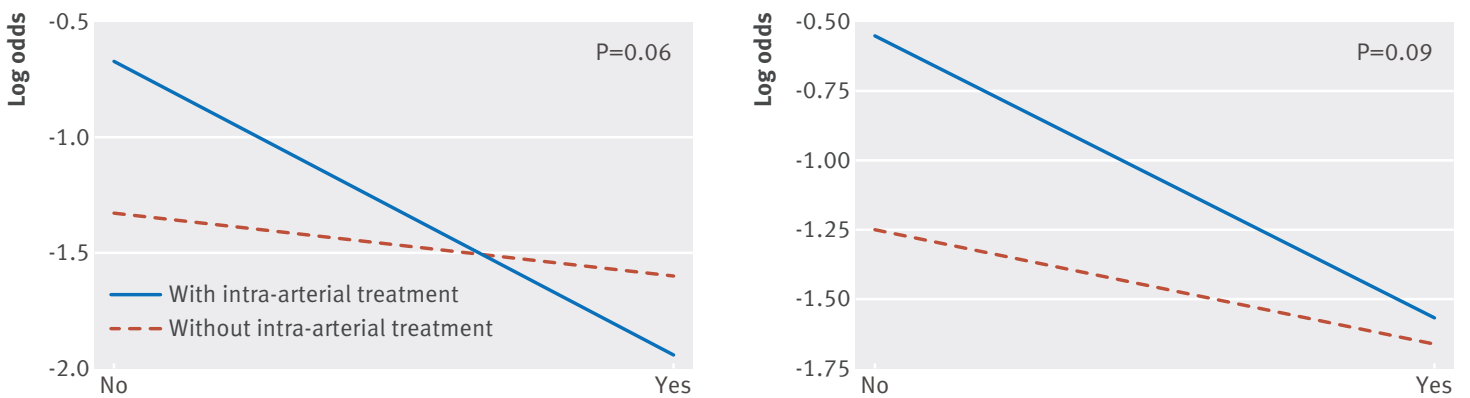

Previous stroke

Atrial fibrillation
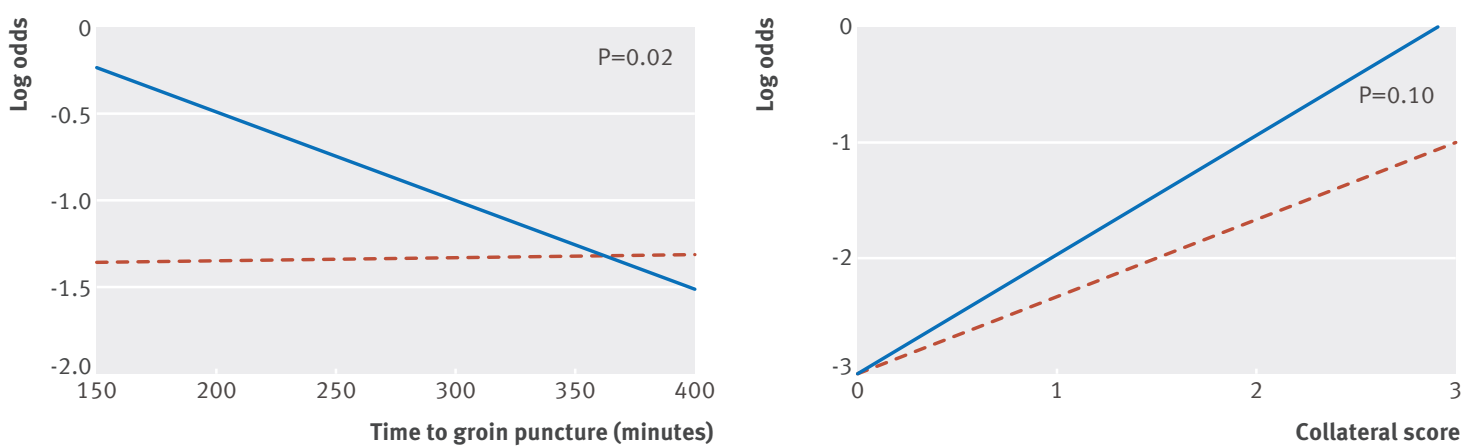

Collateral score

Fig 1 | Univariable interaction effects in derivation cohort $(n=500)$. Interaction with treatment is expressed as log odds for good functional outcome (modified Rankin Scale score 0-2) with and without intra-arterial treatment on the y axis.

Variables on $x$ axis are expressed continuously (time to groin puncture) or categorically (previous stroke, atrial fibrillation, and collateral score)

We implemented our model in a web application that provides predictions of outcome for individual patients with acute ischaemic stroke based on baseline clinical and radiological characteristics for use in clinical practice. It shows bar charts with the expected distribution of mRS categories with and without intra-arterial treatment, the predicted probabilities of good functional outcome, and the predicted absolute treatment benefit (fig 4). This web application was made accessible online at www.mrpredicts.com. 


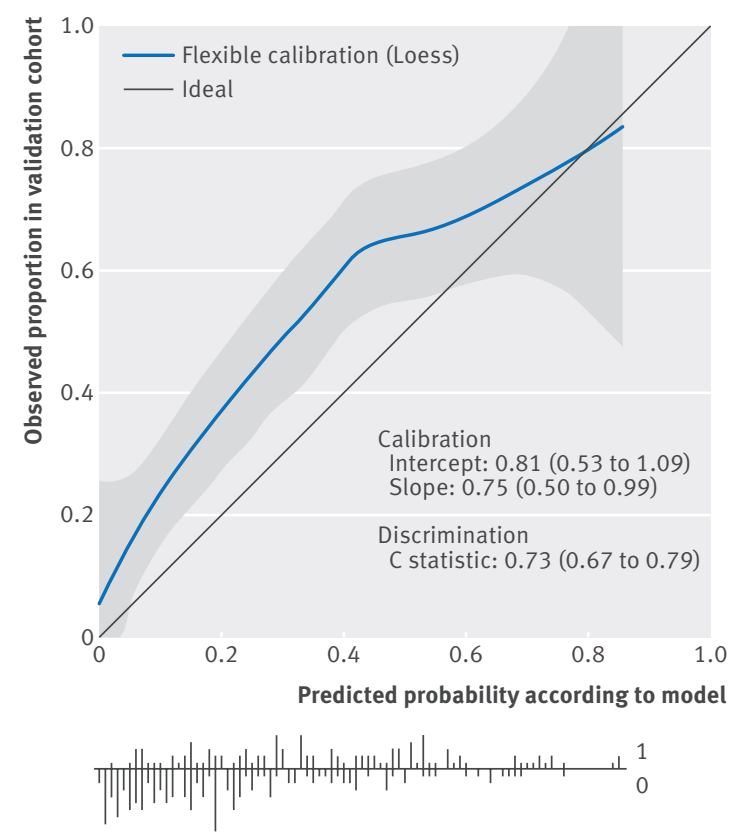

Fig 2 | Calibration plot for predicted good functional outcome, defined as modified Rankin Scale (mRS) score $0-2$, in validation cohort $(\mathrm{n}=260)$. The calibration slope reflects the strength of predictors. The calibration intercept reflects the calibration in the large, indicating whether predicted probabilities are systematically too low or too high. The overall observed proportion of patients with $\mathrm{mRS}$ score $0-2$ in the validation cohort was higher as to be expected using our model. The linear bar chart shows the distribution of patients with $(=1)$ or without $(=0)$ an observed outcome of mRS score 0 -2. Discrimination between low and high likelihood of good functional outcome was moderate ( $C$ statistic $0.73,95 \%$ confidence interval 0.67 to 0.79 )

\section{Discussion}

We developed and externally validated a clinical decision tool to predict the benefit of intra-arterial treatment for individual patients with acute ischaemic stroke, based on multiple patient characteristics. The predicted treatment benefit varied substantially between individual patients with different risk profiles.

\section{Strengths and weaknesses in relation to other studies}

Two risk scores have been described previously for the prediction of functional outcome after intra-arterial treatment. ${ }^{2728}$ These scores are of limited value because they were developed on older cohorts of patients who were treated before the introduction of stent retrievers and contain only a small number of clinical variables. Furthermore, they do not provide individual predictions and most of the variables and outcome measures in these studies had been dichotomised, which is considered to be statistically inefficient and biologically implausible. ${ }^{29}$ Our model combines 11 baseline clinical and radiological characteristics simultaneously to provide individualised predictions of the effect of intra-arterial treatment. In contrast, conventional subgroup analyses focus mainly on predictive effects and assess the effect of only one variable at a time. Previous subgroup analyses of trials on intra-arterial treatment have tested whether there are differences in effect of such treatment based on time to treatment, ${ }^{30-34}$ stroke severity, ${ }^{1235}$ and collateral score. ${ }^{15}$ Analysing one variable at a time may provide mechanistic insights to inform future studies and shape clinical considerations. However, they are of limited value in individual patient care, because treatment benefit is influenced by multiple individual factors simultaneously. ${ }^{13} 14$ Furthermore, even with similar relative treatment effects, individual patients may have different absolute treatment effects owing to different baseline risks. More targeted individual treatment decisions can be obtained by using a more complex multivariable modelling approach to identify individual patients with large or small expected treatment benefit. ${ }^{13}$

We found modest interaction with treatment for history of ischaemic stroke, collateral score, and time from stroke onset to groin puncture. For collateral score and time to groin puncture, interaction with effect of intra-arterial treatment was already shown in previous subgroup analyses. ${ }^{1530}$ Both variables are clinically likely to cause an interaction with intra-arterial treatment. However, previous stroke has not been studied for interaction with treatment before, and was an unexpected finding in our study. It may be a chance finding, since it was not reproduced in the validation cohort and we have no clinical explanation. When the regression coefficients were fitted on data of the combined derivation and validation cohort and the coefficients of interaction terms were reduced to prevent overfitting, the interaction effect for previous stroke in the final model was small. Further validation should reveal whether the relative effect of intra-arterial treatment is modified by experience of a previous stroke.

Our study has several limitations. The discriminative ability of the model in the external validation was modest. It should be emphasised that the $\mathrm{C}$ statistic for the ordinal outcome is a conservative measure. It assesses discrimination between exact categories of the mRS, instead of discrimination between two groups with different outcome (eg, mRS score 0-2 $v$ mRS score 3-6). Externally validated C statistics of all cut-offs were better than the ordinal C statistic (eg, 0.73 for good functional outcome and 0.75 for mortality). Nevertheless, the relatively small sample size and inclusion of interaction terms in the model may have resulted in some optimism and overfitting, despite shrinkage of the regression coefficients. The calibration was also suboptimal; despite the fact that most patients were treated with first generation thrombectomy devices, patients in the Interventional Management of Stroke III trial (IMS III) had a better outcome than predicted by our model. This could be explained by the patient selection in IMS III (eg, premorbid mRS score $0-2$, age $<82$ years, treatment with intravenous tissue plasminogen activator), ${ }^{36}$ which resulted in a better prognosis overall. Patients in the 
IMS III control group had better outcomes than patients in the control group in the Multicenter Randomised Clinical Trial of Endovascular Treatment for Acute Ischaemic Stroke in the Netherlands (MR CLEAN) trial (mRS score 0-2=39\% (IMS III with occlu-
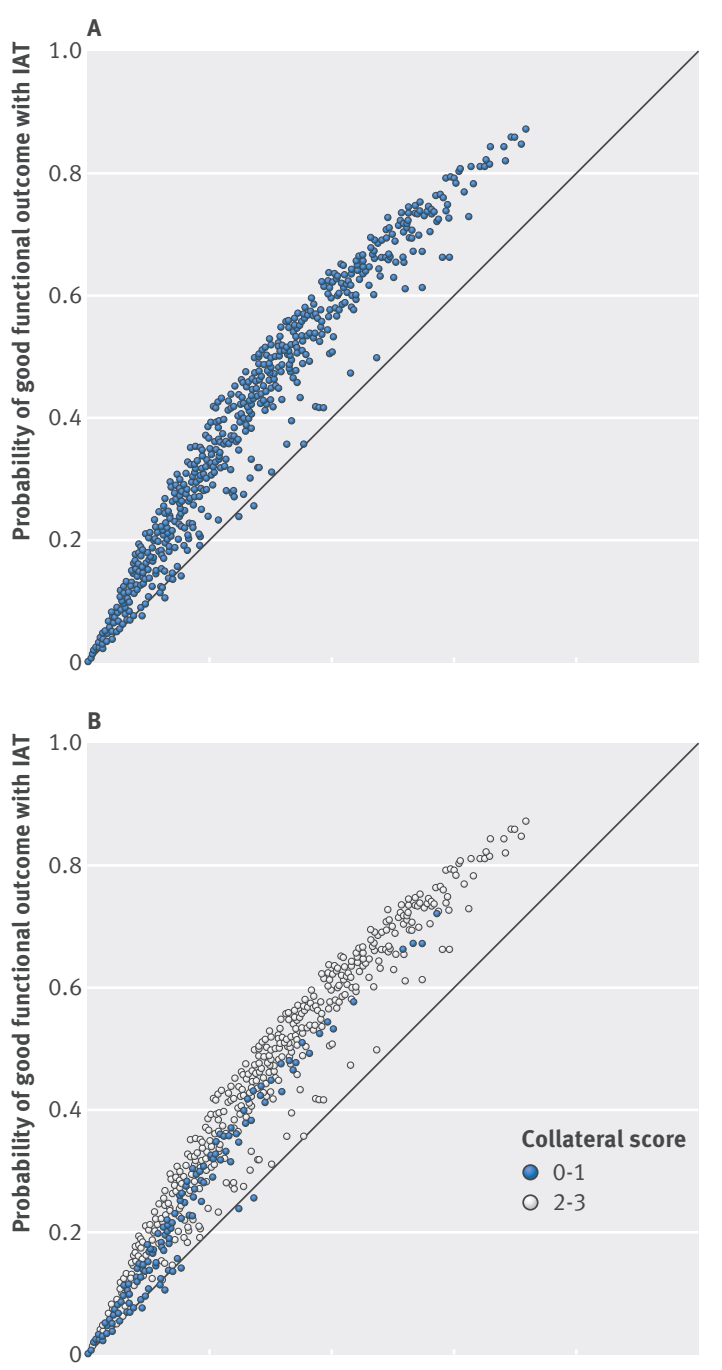

Fig 3 | (A) Predicted probabilities of good functional outcome (modified Rankin Scale (mRS) score 0-2) for all individual patients in combined derivation and validation cohort $(\mathrm{n}=760)$. Each dot represents one individual patient, with the probability of good functional outcome (mRS score 0-2) without intra-arterial treatment (IAT) expressed on $x$ axis, and probability for good functional outcome with IAT on y axis. Above the diagonal line the predicted probability of good functional outcome with IAT is higher than that without IAT. The farther above this line, the larger the predicted effect of treatment. (B) Patients highlighted with no or poor collaterals (score $0-1$ ). (C) Patients highlighted with low Alberta Stroke Program Early CT score (ASPECTS, score 0 -5) sion on CT angiography) $v$ 19\% (MR CLEAN)), leading to inadequate calibration of our model. ${ }^{536}$

\section{Implications for clinicians}

Despite its limitations, the currently developed model is the first to predict the effect of intra-arterial treatment for individual patients on arrival at the emergency department. When compared with other models used in neurovascular practice, HAS-BLED (C statistic 0.65) and $\mathrm{CHA}_{2} \mathrm{DS}_{2}$-VASc (0.61), it performs accurately. ${ }^{3738}$ The predictions made by our decision tool often agree with clinical intuition, which should not be surprising. However, estimates derived from large datasets are preferable to the subjective opinion of a doctor, whose experience, no matter how vast, can never match the information contained in large datasets. ${ }^{39}$

Currently, some centres withhold intra-arterial treatment in specific subgroups of patients (eg, low ASPECTS, no collaterals, age $>80$ years, or M2 occlusion). Indeed, our model predicts no benefit of intra-arterial treatment for some patients, especially when a patient has more than one characteristic that negatively affects the effect of intra-arterial treatment. The decision not to treat may be particularly relevant in patients who have to be transferred to an intervention centre. The model may help to identify patients without expected benefit of intra-arterial treatment and topple the balance in favour of no treatment. More importantly, our study shows that treatment should not be withheld based on one characteristic. Some patients belonging to one of the subgroups that are considered as having no benefit of intra-arterial treatment, such as poor collaterals or low ASPECTS, may still benefit from intra-arterial treatment substantially if other characteristics are favourable. This emphasises the importance of making personalised treatment decisions, instead of using average treatment effects, and shows the need for combining multiple clinical and radiological baseline characteristics instead of withholding treatment based on one characteristic. ${ }^{40}$

This is the first model for intra-arterial treatment decision making. The predictions of our model should be considered as a starting point for clinical decision making, and not as a final recommendation. Our model was developed using the MR CLEAN database, consisting of an unselected population with few selection criteria. Therefore, our model is likely applicable in centres that use few clinical and radiological selection criteria. Future analyses within larger studies may refine the current recommendations and improve the validity of the model.

\section{Conclusion}

The proposed clinical decision tool combines multiple baseline clinical and radiological characteristics and shows large variations in treatment benefit between patients. The tool is clinically useful as it aids in identifying individual patients who may benefit from intra-arterial treatment for acute ischaemic stroke. 
Clinical decision tool for intra-arterial treatment in acute ischaemic stroke

\begin{tabular}{|c|c|}
\hline \multicolumn{2}{|c|}{ Patient characteristics } \\
\hline Age (years) & ASPECT score \\
\hline 70 & (0) \\
\hline NIHSS & \multirow{4}{*}{$\begin{array}{l}\text { Location of occlusion } \\
\text { Intracranial ICA (with } \\
\text { or without M1) } \\
\text { M1 only } \\
\text { O M2 only }\end{array}$} \\
\hline 22 & \\
\hline Pre-stroke mRS & \\
\hline 0 & \\
\hline Previous stroke & \multirow{4}{*}{$\begin{array}{l}\text { CTA collateral score } \\
\odot 0 \text { - absent collaterals } \\
01 \text { - poor ( }(50 \% \text { filling) } \\
02 \text { - moderate ( }>50 \% \text { filling) } \\
\text { 3 - good collaterals }\end{array}$} \\
\hline No & \\
\hline Diabetes mellitus & \\
\hline No & \\
\hline $\begin{array}{l}\text { Systolic blood } \\
\text { pressure (mm Hg) }\end{array}$ & \multirow{3}{*}{$\begin{array}{l}\text { Estimated time from } \\
\text { onset to groin puncture } \\
\text { (minutes) }\end{array}$} \\
\hline 140 & \\
\hline Intravenous tPA & \\
\hline Yes & 100 \\
\hline
\end{tabular}

Patient 2

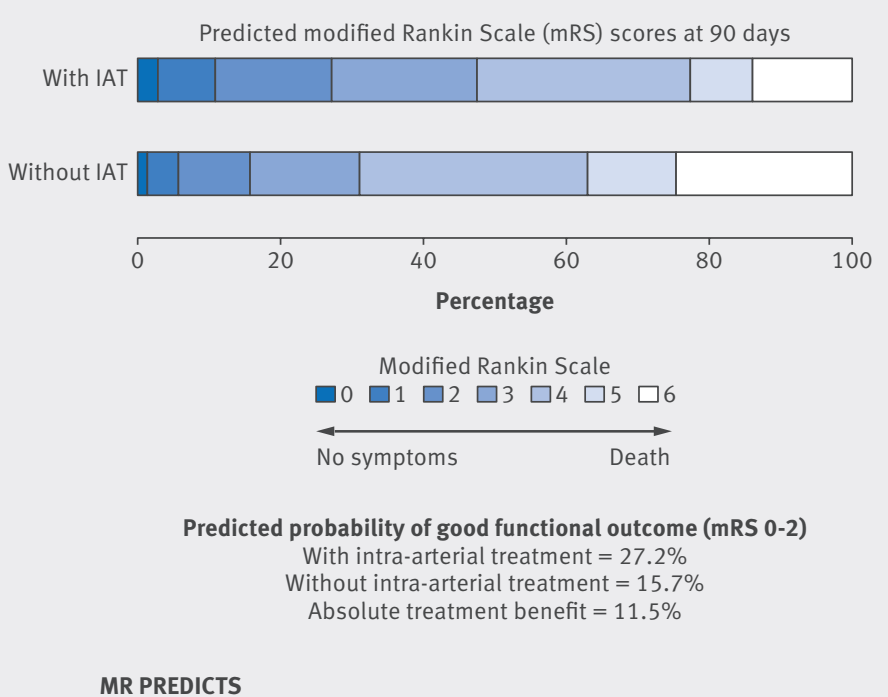

MR PREDICTS

Clinical decision tool for intra arterial treatment in acute ischaemic stroke

\begin{tabular}{|c|c|}
\hline \multicolumn{2}{|c|}{ Patient characteristics } \\
\hline Age (years) & ASPECT score \\
\hline 80 & (0) \\
\hline NIHSS & \multirow{4}{*}{$\begin{array}{l}\text { Location of occlusion } \\
\odot \text { Intracranial ICA (with } \\
\text { or without M1) } \\
\text { M1 only } \\
\text { M2 only }\end{array}$} \\
\hline 22 & \\
\hline Pre-stroke mRS & \\
\hline 0 & \\
\hline Previous stroke & \multirow{4}{*}{$\begin{array}{l}\text { CTA collateral score } \\
\text { O } 0 \text { - absent collaterals } \\
\text { o } 1 \text { - poor ( }(50 \% \text { filling) } \\
\text { 2 } \text { - moderate ( }>50 \% \text { filling) } \\
\text { o } 3 \text { - good collaterals }\end{array}$} \\
\hline No & \\
\hline Diabetes mellitus & \\
\hline Yes & \\
\hline $\begin{array}{l}\text { Systolic blood } \\
\text { pressure }(\mathrm{mm} \mathrm{Hg})\end{array}$ & \multirow{3}{*}{$\begin{array}{l}\text { Estimated time from } \\
\text { onset to groin puncture } \\
\text { (minutes) }\end{array}$} \\
\hline 180 & \\
\hline Intravenous tPA & \\
\hline No & 350 \\
\hline
\end{tabular}

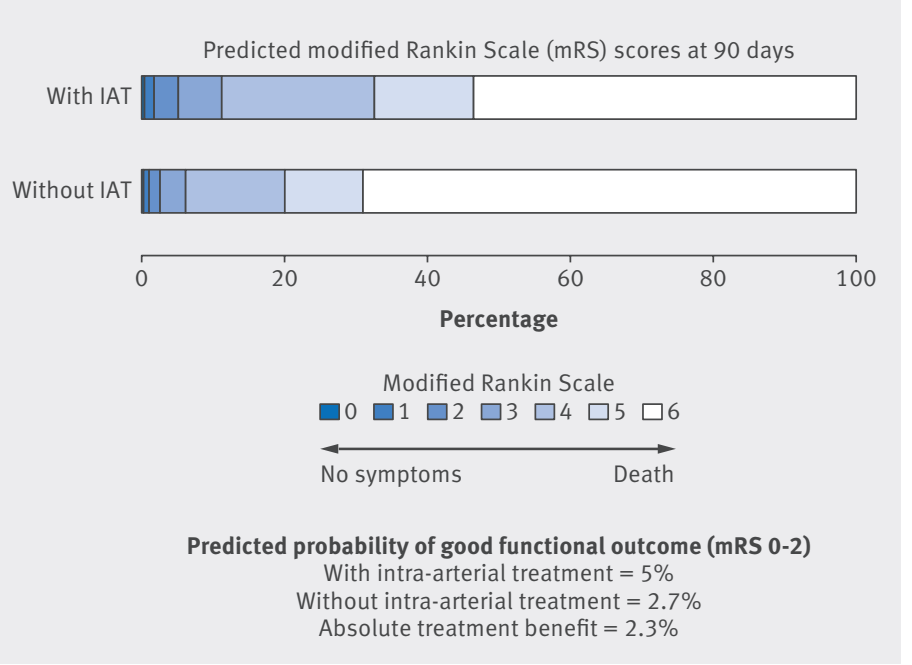

Fig 4 | A stylised representation of the clinical decision tool. Baseline characteristics and predicted probabilities of good functional outcome (modified Rankin Scale (mRS) score 0-2) for two examples (see introduction). ASPECT=Alberta Stroke Program Early Computed Tomography Score; IAT=intra-arterial treatment; NIHSS=National Institutes of Health Stroke Scale; ICA=internal carotid artery; CTA=computed tomography angiography

\section{AUTHOR AFFILIATIONS}

'Department of Public Health, Erasmus MC University Medical Centre Rotterdam, PO Box 2040, 3000 CA Rotterdam, Netherlands 2Department of Neurology, Erasmus MC University Medical Centre Rotterdam, Rotterdam, Netherlands

${ }^{3}$ Department of Radiology, Erasmus MC University Medical Centre Rotterdam, Rotterdam, Netherlands

4Department of Neurology and Rehabilitation Medicine, University of Cincinnati Gardner Neuroscience Institute, Cincinnati, OH, USA

${ }^{5}$ Department of Public Health Sciences, Medical University of South Carolina, Charleston, SC, USA

${ }^{6}$ Department of Radiology, Academic Medical Centre, Amsterdam, Netherlands

${ }^{7}$ Department of Radiology, Maastricht University Medical Centre, Maastricht, Netherlands

${ }^{8}$ Department of Neurology, Academic Medical Centre, Amsterdam, Netherlands
${ }^{9}$ Department of Neurology, Maastricht University Medical Centre, Maastricht, Netherlands

${ }^{10}$ Department of Medical Statistics and Bioinformatics, Leiden University Medical Centre, Leiden, Netherlands

Contributors: BR, HFL, EWS, DWID, BJE, JPB, SDY, PK, OAB, YBWEMR, RJO, WHZ, CBLMM, and AL conceived the study. MJHLM, EV, BR, HFL, EWS, and DWJD designed the study. MJHLM and EV performed the literature search, data analysis, and statistical modelling. They contributed equally to the study. MJHLM, EV, BR, and HFL drafted the manuscript. EWS, DWJD, BJE, JPB, SDY, PK, OAB, YBWEMR, RJO, WHZ, CBLMM, and AL performed a critical review of the manuscript. HFL is the guarantor.

Funding: This study was funded by the Erasmus MC Cost-Effectiveness Research. The Interventional Management of Stroke III (IMS III) trial was funded by National Institutes of Health and National Institute of Neurological Disorders and Stroke grant numbers: University of Cincinnati (U01NS052220) and Medical University of South Carolina (U01NS054630 and U01NS077304). Genentech supplied the study drug used for intra-arterial tissue-type plasminogen activator 
treatment in the endovascular group. EKOS, Concentric Medical, and Cordis supplied study catheters during protocol versions 1 to 3 . In the United States, IMS III investigator meeting support was provided in part by Genentech, EKOS, and Concentric Medical. In Europe, IMS III investigator meeting support was provided in part by Boehringer Ingelheim. The Multicenter Randomised Clinical Trial of Endovascular Therapy for Acute Ischaemic Stroke in the Netherlands (MR CLEAN) was partly funded by the Dutch Heart Foundation and by unrestricted grants from Angiocare BV, Covidien, medac, Lamepro Benelux, Penumbra, Stryker, TOP Medical, and Concentric Medical.

Competing interests: All authors have completed the ICMJE uniform disclosure form. JPB received study medication for intra-arterial tissue type plasminogen activator from Genentech and catheters were supplied by EKOS Corporation, Concentric Medical, and Cordis. His research is funded by the Department of Neurology and Rehabilitation Medicine. He is remunerated by Genentech for his role on the Steering Committee for A Study of the Efficacy and Safety of Activase (Alteplase) in Patients With Mild Stroke (PRISMS) trial. SDY is remunerated by Genentech for her statistical role in the PRISMS trial. PK is remunerated by Genentech for her role as lead principal investigator of the PRISMS trial and by Penumbra for her role as neurology principal investigator of the Assess the Penumbra System in the Treatment of Acute Stroke trial. She has also received royalties from UpToDate and consulted for Grand Rounds, St Jude Medical, and Biogen. BJE is remunerated for his role as CE mark reviewer by DEKRA and by Novartis for educational lectures. Erasmus $M C$ received funds from Stryker for consultations by DWJD and AL and for training courses by BJE, and from Bracco Imaging for consultations by DWJD. The Academic Medical Centre received funds from Stryker for consultations by CBLMM and YBWEMR. Maastricht University Medical Centre received funds from Stryker and Codman for consultations by WHZ.

Ethical approval: Not required.

Data sharing: No additional data available.

Transparency: The lead authors (MJHLM and EV) affirm that the manuscript is an honest, accurate, and transparent account of the study being reported; that no important aspects of the study have been omitted; and that any discrepancies from the study as planned have been explained.

This is an Open Access article distributed in accordance with the Creative Commons Attribution Non Commercial (CC BY-NC 4.0) license, which permits others to distribute, remix, adapt, build upon this work non-commercially, and license their derivative works on different terms, provided the original work is properly cited and the use is non-commercial. See: http://creativecommons.org/licenses/ by-nc/4.0/.

1 Lopez AD, Mathers CD, Ezzati M, Jamison DT, Murray CJ. Global and regional burden of disease and risk factors, 2001: systematic analysis of population health data. Lancet 2006;367:1747-57. doi:10.1016/S0140-6736(06)68770-9.

2 van der Worp HB, van Gijn I. Clinical practice. Acute ischemic stroke. N Engl J Med 2007;357:572-9. doi:10.1056/NEJMcp072057.

3 Smith WS, Lev MH, English JD, et al. Significance of large vessel intracranial occlusion causing acute ischemic stroke and TIA. Stroke 2009;:40:3834-40. doi:10.1161/STROKEAHA.109.561787.

4 Lima FO, Furie KL, Silva GS, et al. Prognosis of untreated strokes due to anterior circulation proximal intracranial arterial occlusions detected by use of computed tomography angiography. JAMA Neurol 2014;71:151-7. doi:10.1001/jamaneurol.2013.5007.

5 Berkhemer OA, Fransen PS, Beumer D, et al. MR CLEAN Investigators. A randomized trial of intraarterial treatment for acute ischemic stroke. N Engl/ Med 2015:372:11-20. doi:10.1056/NEJMoa1411587.

6 Campbell BC, Mitchell PJ, Kleinig TJ, et al. EXTEND-IA Investigators. Endovascular therapy for ischemic stroke with perfusion-imaging selection. N Engl / Med 2015;372:1009-18. doi:10.1056/ NEJMoa1414792.

7 Goyal M, Demchuk AM, Menon BK, et al. ESCAPE Trial Investigators. Randomized assessment of rapid endovascular treatment of ischemic stroke. N Engl J Med 2015;372:1019-30. doi:10.1056/ NEJMoa1414905.

8 Jovin TG, Chamorro A, Cobo E, et al. REVASCAT Trial Investigators. Thrombectomy within 8 hours after symptom onset in ischemic stroke. N Engl J Med 2015;372:2296-306. doi:10.1056/ NEJMoa1503780.

9 Saver JL, Goyal M, Bonafe A, et al. SWIFT PRIME Investigators. Stent-retriever thrombectomy after intravenous t-PA vs. t-PA alone in stroke. N Engl J Med 2015;372:2285-95. doi:10.1056/ NEJMoa1415061.

10 Bracard S, Ducrocq X, Mas JL, et al. THRACE investigators. Mechanica thrombectomy after intravenous alteplase versus alteplase alone after stroke (THRACE): a randomised controlled trial. Lancet Neurol 2016;15:1138-47. doi:10.1016/S1474-4422(16)30177-6.
11 Muir KW, Ford GA, Messow CM, et al. PISTE Investigators. Endovascular therapy for acute ischaemic stroke: the Pragmatic Ischaemic Stroke Thrombectomy Evaluation (PISTE) randomised, controlled trial. J Neurol Neurosurg Psychiatry 2017;88:38-44. doi:10.1136/jnnp-2016-314117.

12 Goyal M, Menon BK, van Zwam WH, et al. HERMES collaborators. Endovascular thrombectomy after large-vessel ischaemic stroke: a meta-analysis of individual patient data from five randomised trials. Lancet 2016;387:1723-31. doi:10.1016/S0140-6736(16)00163-X.

13 Kent DM, Rothwell PM, Ioannidis JP, Altman DG, Hayward RA. Assessing and reporting heterogeneity in treatment effects in clinical trials: a proposal. Trials 2010;11:85. doi:10.1186/1745-6215 $11-85$

14 Rothwell PM. Treating individuals 2. Subgroup analysis in randomised controlled trials: importance, indications, and interpretation. Lancet 2005:365:176-86. doi:10.1016/S0140-6736(05)17709-5.

15 Berkhemer OA, Jansen IG, Beumer D, et al. MR CLEAN Investigators. Collateral Status on Baseline Computed Tomographic Angiography and Intra-Arterial Treatment Effect in Patients With Proximal Anterior Circulation Stroke. Stroke 2016:47:768-76.

16 Barber PA, Demchuk AM, Zhang J, Buchan AM. Validity and reliability of a quantitative computed tomography score in predicting outcome of hyperacute stroke before thrombolytic therapy. ASPECTS Study Group. Alberta Stroke Programme Early CT Score. Lancet 2000;355:1670-4

17 Tan IYL, Demchuk AM, Hopyan J, et al. CT angiography clot burden score and collateral score: correlation with clinical and radiologic outcomes in acute middle cerebral artery infarct. Am J Neuroradiol 2009;30:525-31. doi:10.3174/ajnr.A1408.

18 Fransen PS, Beumer D, Berkhemer OA, et al. MR CLEAN Investigators. MR CLEAN, a multicenter randomized clinical trial of endovascular treatment for acute ischemic stroke in the Netherlands: study protocol for a randomized controlled trial. Trials 2014;15:343. doi:10.1186/1745-6215-15-343

19 Mulder MJHL, Venema E, Roozenbeek B, et al. Towards personalised intra-arterial treatment of patients with acute ischaemic stroke: a study protocol for development and validation of a clinical decision aid. BMJ Open 2017:7:e013699.

20 van Swieten JC, Koudstaal PJ, Visser MC, Schouten HJ, van Gijn J. Interobserver agreement for the assessment of handicap in stroke patients. Stroke 1988;19:604-7. doi:10.1161/01.STR.19.5.604.

21 Harrell FE. Regression modeling strategies: with applications to linear models, logistic regression, and survival analysis. Springer-Verlag, 2001doi:10.1007/978-1-4757-3462-1.

22 Steyerberg EW. Clinical prediction models: a practical approach to development, validation, and updating.Springer 2009doi:10.1007/978-0-387-77244-8.

23 Broderick JP, Palesch YY, Demchuk AM, et al. Interventional Management of Stroke (IMS) III Investigators. Endovascular therapy after intravenous t-PA versus t-PA alone for stroke. N Engl / Med 2013:368:893-903. doi:10.1056/NEIMoa1214300.

24 Khatri P, Hill MD, Palesch YY, et al. Interventional Management of Stroke III Investigators. Methodology of the Interventional Management of Stroke III Trial. Int J Stroke 2008;3:130-7. doi:10.1111/j.1747-4949.2008.00151.x

25 Steyerberg EW, Vickers AJ, Cook NR, et al. Assessing the performance of prediction models: a framework for traditional and novel measures. Epidemiology 2010;21:128-38. doi:10.1097/ EDE.0b013e3181c30fb2.

26 Steyerberg EW, Vergouwe Y. Towards better clinical prediction models: seven steps for development and an ABCD for validation. Eur Heart J 2014:35:1925-31. doi:10.1093/eurhearti/ehu207.

27 Hallevi H, Barreto AD, Liebeskind DS, et al. UCLA Intra-Arterial Therapy Investigators. Identifying patients at high risk for poor outcome after intra-arterial therapy for acute ischemic stroke. Stroke 2009:40:17805. doi:10.1161/STROKEAHA.108.535146.

28 Flint AC, Cullen SP, Faigeles BS, Rao VA. Predicting long-term outcome after endovascular stroke treatment: the totaled health risks in vascular events score. A/NR Am / Neuroradiol 2010;31:1192-6. doi:10.3174/ajnr.A2050.

29 Scott SC, Goldberg MS, Mayo NE. Statistical assessment of ordinal outcomes in comparative studies. / Clin Epidemiol 1997;50:45-55. doi:10.1016/S0895-4356(96)00312-5

30 Fransen PS, Berkhemer OA, Lingsma HF, et al. Time to Reperfusion and Treatment Effect for Acute Ischemic Stroke: A Randomized Clinical Trial. JAMA Neurol 2015;73:1-7.

31 Goyal M, Jadhav AP, Bonafe A, et al. SWIFT PRIME investigators. Analysis of Workflow and Time to Treatment and the Effects on Outcome in Endovascular Treatment of Acute Ischemic Stroke: Results from the SWIFT PRIME Randomized Controlled Trial. Radiology 2016;279:888-97. doi:10.1148/radiol.2016160204

32 Menon BK, Sajobi TT, Zhang Y, et al. Analysis of Workflow and Time to Treatment on Thrombectomy Outcome in the Endovascular Treatment for Small Core and Proximal Occlusion Ischemic Stroke (ESCAPE) Randomized, Controlled Trial. Circulation 2016;133:2279-86. doi:10.1161/CIRCULATIONAHA.115.019983. 
33 Ribo M, Molina CA, Cobo E, et al. REVASCAT Trial Investigators. Association Between Time to Reperfusion and Outcome Is Primarily Driven by the Time From Imaging to Reperfusion. Stroke 2016:47:9991004. doi:10.1161/STROKEAHA.115.011721.

34 Saver JL, Goyal M, van der Lugt A, et al. HERMES Collaborators. Time to treatment with endovascular thrombectomy and outcomes from ischemic stroke: a meta-analysis. JAMA 2016;316:1279-88.

35 Broderick JP, Berkhemer OA, Palesch YY, et al. IMS III Investigators MR CLEAN Investigators. Endovascular Therapy Is Effective and Safe for Patients With Severe Ischemic Stroke: Pooled Analysis of Interventional Management of Stroke III and Multicenter Randomized Clinical Trial of Endovascular Therapy for Acute Ischemic Stroke in the Netherlands Data. Stroke 2015;46:3416-22.

36 Demchuk AM, Goyal M, Yeatts SD, et al. IMS III Investigators. Recanalization and clinical outcome of occlusion sites at baseline CT angiography in the Interventional Management of Stroke III trial. Radiology 2014;273:202-10. doi:10.1148/radiol.14132649.
37 Lip GY, Frison L, Halperin JL, Lane DA. Comparative validation of a novel risk score for predicting bleeding risk in anticoagulated patients with atrial fibrillation: the HAS-BLED (Hypertension, Abnormal Renal/ Liver Function, Stroke, Bleeding History or Predisposition, Labile INR, Elderly, Drugs/Alcohol Concomitantly) score. J Am Coll Cardiol 2011:57:173-80. doi:10.1016/j.jacc.2010.09.024.

38 Lip GY, Nieuwlaat R, Pisters R, Lane DA, Crijns HJ. Refining clinical risk stratification for predicting stroke and thromboembolism in atrial fibrillation using a novel risk factor-based approach: the euro heart survey on atrial fibrillation. Chest 2010:137:263-72. doi:10.1378/chest.09-1584.

39 Lingsma HF, Roozenbeek B, Steyerberg EW, Murray GD, Maas Al. Early prognosis in traumatic brain injury: from prophecies to predictions. Lancet Neurol 2010;9:543-54. doi:10.1016/S1474-4422(10)70065-X.

40 Kent DM, Nelson J, Dahabreh IJ, Rothwell PM, Altman DG, Hayward RA. Risk and treatment effect heterogeneity: re-analysis of individual participant data from 32 large clinical trials[published online ahead of print July 3, 2016]. Int JEpidemiol 2016;dyw118. doi:10.1093/ije/dyw118. 